\title{
RELAÇÃO ENTRE CARACTERÍSTICAS PSICOLÓGICAS E O DESEMPENHO FÍSICO DE ACADÊMICOS DE EDUCAÇÃO FÍSICA
}

\section{Relation between psychological characteristics and the physical performance of physical education academics}

\author{
Érico Felden Pereira', Clarissa Stefani Teixeira², Maria Cristina Chimelo Paim ${ }^{3}$ \\ 1 Universidade Federal do Paraná - Curitiba - PR - Brasil. \\ ${ }^{2}$ Universidade Federal de Santa Catarina - Florianópolis - SC - Brasil. \\ ${ }^{3}$ Universidade Luterana do Brasil - Santa Maria - RS - Brasil. \\ ${ }^{3}$ Núcleo de Estudos do Movimento Humano - Universidade Federal de Lavras - Lavras - MG - Brasil.
}

\begin{abstract}
Resumo: O objetivo deste estudo foi identificar a relação entre as peculiaridades tipológicas do sistema nervoso, os traços de personalidade e o desempenho físico de acadêmicos de Educação Física. A amostra foi formada por 29 indivíduos, 14 do gênero masculino (19,36 $\pm 2,06$ anos) e 15 do gênero feminino $(20,53 \pm 2,97)$, todos acadêmicos ingressantes no curso de Educação Física. Foram realizados testes físicos para avaliação da resistência muscular localizada, resistência aeróbica, flexibilidade, potência de membros inferiores e superiores, velocidade e agilidade. As peculiaridades tipológicas do sistema nervoso e dos traços de personalidade foram avaliados por meio de questionários. Os resultados demonstraram que os acadêmicos possuem níveis regulares de aptidão física e características psicológicas diferenciadas da população em geral. Não foram encontradas correlações estatisticamente significativas entre os índices das peculiaridades tipológicas do sistema nervoso e dos traços de personalidade com o desempenho na maioria dos testes físicos realizados.
\end{abstract}

Palavras-chave: Peculiaridades Tipológicas do Sistema Nervoso, Traços de Personalidade, Desempenho Físico.

Abstract: This study aimed to investigate the relationship between the typological peculiarities of the nervous system, personality traces and the physical performance of physical education academics. The sample was constituted of 29 individuals, 14 males $(19,36 \pm 2,06$ years $)$ and 15 females $(20,53 \pm 2,97)$, all freshmen of Physical Education. A battery of physical tests was carried out to evaluate localized muscular resistance, aerobic endurance, flexibility, muscular power of the inferior and superior limbs, speed and agility. The peculiarities of the nervous system and personality traces were evaluated by means of questionnaires. The results indicated that the academics have a regular physical performance and psychological characteristics which differ from the population in general. There were no statistical correlations between the indices of peculiarities of the nervous system and personality traces with performance in most physical tests.

Key words: Peculiarities of the Nervous System, Personality Traces and Physical Performance.

Aceito em 27/10/2008 - Rev. Educ. Fís. 2009 Mar: 144: 3-12. Rio de Janeiro - RJ - Brasil

\section{INTRODUÇÃO}

Estudiosos têm buscado esclarecer as dimensões que definem as diferenças individuais das pessoas, especialmente no que tange à personalidade. De forma geral, a interação entre fatores biológicos e ambientais molda o comportamento individual e, especificamente, em relação à base biológica do temperamento, os mecanismos anotomo-fisiológicos, bioquímicos e de hereditariedade são alguns dos parâmetros de análise (1).
A personalidade, segundo Allport ${ }^{(2)}$, pode ser vista como a organização dinâmica dos elementos físicos e psíquicos que definem os comportamentos característicos de cada indivíduo; já o temperamento é considerado a base emocional da personalidade. Duas teorias podem ser destacadas na análise da personalidade: a Teoria do Temperamento de Pavlov (3) que liga os tipos de temperamento à atividade do sistema nervoso central e considera a reação (excitação ou inibição) das pessoas às diferentes situações da vida para definir o temperamento e, a Teoria de Eysenck ${ }^{(4)}$, que considera os traços extroversão e 
neurotismo como as duas dimensões primárias da personalidade.

O construto personalidade é permeado por inúmeros fatores que se estruturam e se relacionam entre si de maneira única em cada pessoa e contribuem para determinar aspectos importantes do indivíduo, sua forma de agir, seu desempenho em tarefas diárias, sua saúde e sua motivação para determinadas atividades ${ }^{(5)}$. Da mesma forma, o desenvolvimento da aptidão física geral também está ligado à interação entre as características biológicas, psíquicas e culturais e é possível que grupos específicos de pessoas possuam uma tendência a apresentar características individuais, tanto físicas como de comportamento, semelhantes ${ }^{(6)}$.

Pesquisas com acadêmicos de educação física identificaram traços de personalidade diferenciados considerando a população geral ${ }^{(7,8)}$. No entanto, análises da aptidão física nesta mesma população indicaram desempenho relativo baixo em testes de desempenho físico ${ }^{(9)}$. O perfil do profissional de educação física, conforme discutem Pereira e Graup ${ }^{(9)}$, nos últimos anos vêm passando de um estereótipo de profissional essencialmente prático, com o objetivo de atender as necessidades exclusivamente físicas dos indivíduos por meio do culto ao corpo e aquisição de habilidades motoras, para um profissional com perfil de formação ampliada com capacidade de considerar os avanços científicos da área e as demandas cada vez maiores da profissão.

Neste contexto, objetivou-se neste estudo identificar a relação entre as peculiaridades tipologias do sistema nervoso, os índices dos traços de personalidade extroversão/introversão e instabilidade/ estabilidade emocional e o desempenho físico em acadêmicos de Educação Física.

\section{METODOLOGIA}

A amostra foi formada por 29 acadêmicos, sendo 14 do gênero masculino e 15 do gênero feminino ingressantes no curso de graduação em Educação Física de uma instituição federal do sul do Brasil. A seleção de amostra, de forma intencional, foi realizada da seguinte forma: convite a todos os acadêmicos ingressantes no curso (aproximadamente 40 alunos) sendo a amostra final formada pelos acadêmicos que aceitaram participar do estudo, que realizaram todas as avaliações e que não possuíam restrições para esforço físico nos momentos das coletas. Os acadêmicos que aceitaram participar do estudo assinaram um Termo de Consentimento Livre e Esclarecido contendo informações sobre os procedimentos gerais da pesquisa. Análise estatística descritiva com média e desvio padrão dos índices dos testes físicos e da análise psicológica foi realizada. O teste de correlação de Pearson foi utilizado para verificar a correlação entre o desempenho nos testes físicos e os índices da avaliação psicológica.

Foram realizados os seguintes testes físicos utilizando os respectivos protocolos: "abdominal modificado" (10) - para avaliar resistência muscular localizada, "12 minutos" (11) - para avaliar resistência aeróbica, "sentar e alcançar" (10) - para avaliar flexibilidade, "impulsão horizontal" (12) - para avaliar potência de membros inferiores, "50 metros" (11) _ para avaliar velocidade, "vai e vem" (12) - para avaliar agilidade e "arremesso de medicine-ball" (13) - para avaliar potência de membros superiores.

O "questionário de Strelau" foi aplicado para o diagnóstico das peculiaridades tipológicas do sistema nervoso: força dos processos de excitação; força dos processos de inibição; mobilidade e equilíbrio dos processos. O "questionário de Strelau" se destacou, entre outras metodologias, pelo seu grau de fidedignidade $r>0,9$, objetividade $r>0,9$, e validade $r>0,9$, para $p<0,05$. Este instrumento permite ao pesquisador discriminar os seres humanos por temperamentos: sanguíneos, coléricos, fleumáticos e melancólicos ${ }^{(14)}$. A validação para a língua portuguesa, do "questionário de Strelau", se realizou mediante a aplicação da versão russa e da versão portuguesa 
5 Rev. Educ. Fís. 2009 Mar: 144: 3-12. Rio de Janeiro - RJ - Brasil

em 11 pessoas bilíngües. O coeficiente de correlação RESULTADOS entre as versões em português e em russo foi $r=0,94$ com $\mathrm{p}<0,001^{(16)}$.

Para o diagnóstico dos traços de personalidade extroversão/introversão e instabilidade/estabilidade emocional, utilizou-se o "questionário de Eysenck". A validação para a língua portuguesa, do "questionário de Eysenck", realizou-se mediante a aplicação da versão russa e da versão portuguesa em 10 pessoas bilíngües e o coeficiente de correlação foi de $r=0,92$ com $p<0,001$ na comparação das duas versões ${ }^{(15)}$.

Os resultados dos testes de desempenho físico foram apresentados na TABELA 1 e da identificação dos índices das peculiaridades tipológicas do sistema nervoso e dos traços de personalidade dos acadêmicos nas TABELAS 2 e 3. Nos QUADROS 1,2 e 3 foram apresentados os resultados das análises de correlação entre as variáveis físicas e psicológicas dos acadêmicos, que mostraram relações estatisticamente não significativas nas variáveis investigadas.

TABELA 1

VALORES DO DESEMPENHO NOS TESTES FÍSICOS.

\begin{tabular}{|c|c|c|}
\hline \multirow{2}{*}{ Qualidades físicas } & $\begin{array}{l}\text { Masculino } \\
\quad(n=14)\end{array}$ & $\begin{array}{c}\text { Feminino } \\
\qquad(n=15)\end{array}$ \\
\hline & Média $\pm d p$ & Média $\pm d p$ \\
\hline Idade (anos) & $19,36 \pm 2,06$ & $20,53 \pm 2,97$ \\
\hline Resistência aeróbica (m) & $2495,42 \pm 324,23$ & $1868,87 \pm 255,52$ \\
\hline Resistência muscular localizada & $37,14 \pm 5,26$ & $27,93 \pm 8,43$ \\
\hline Flexibilidade (cm) & $29,55 \pm 9,39$ & $35,75 \pm 8,21$ \\
\hline Velocidade (seg) & $7,32 \pm 0,41$ & $9,32 \pm 0,81$ \\
\hline Potência de membros superiores (m) & $5,08 \pm 0,57$ & $2,90 \pm 0,47$ \\
\hline Potência de membros inferiores (m) & $2,05 \pm 0,17$ & $1,45 \pm 0,12$ \\
\hline Agilidade (seg) & $10,28 \pm 0,56$ & $12,11 \pm 0,46$ \\
\hline
\end{tabular}

TABELA 2

PECULIARIDADES TIPOLÓGICAS DO SISTEMA NERVOSO (PTSN)

\begin{tabular}{cccccccccc}
\hline \multirow{2}{*}{ Acadêmicos } & \multicolumn{2}{c}{ FPE } & \multicolumn{2}{c}{ FPI } & \multicolumn{2}{c}{ MOB } & \multicolumn{2}{c}{ EQU } \\
\cline { 2 - 9 } & Masc & Fem & Masc & Fem & Masc & Fem & Masc & Fem \\
\hline Média & 62,57 & 57,33 & 65,00 & 57,06 & 59,57 & 59,06 & 0,98 & 1,04 \\
Desvio padrão & 13,67 & 12,27 & 13,38 & 14,37 & 11,24 & 12,21 & 0,17 & 0,27 \\
\hline
\end{tabular}

FPE: Força dos processos de excitação / FPI: Força dos processos de inibição / MOB: Mobilidade / EQU: Equilíbrio 
TABELA 3

TRAÇOS DE PERSONALIDADE

\begin{tabular}{ccccc}
\hline \multirow{2}{*}{ Acadêmicos } & \multicolumn{2}{c}{ EX-IN } & \multicolumn{3}{c}{ I-IEE } \\
\cline { 2 - 5 } & Masc & Fem & Masc & Fem \\
\hline Média & 13,29 & 12,40 & 10,14 & 13,73 \\
Desvio padrão & 3,07 & 3,87 & 4,17 & 4,17 \\
\hline
\end{tabular}

EX-IN: Extroversão-introversão / IEE: Instabilidade-estabilidade emocional

QUADRO 1

CORRELAÇÃO ENTRE AS PTSN (FORÇA DOS PROCESSOS DE EXCITAÇÃO E FORÇA DOS PROCESSOS DE INIBIÇÃO) E OS ESCORES DOS TESTES FÍSICOS.

Força dos Processos de Excitação

\begin{tabular}{ccccc}
\hline \multirow{2}{*}{ Componentes da aptidão física } & \multicolumn{2}{c}{ Homens } & \multicolumn{2}{c}{ Mulheres } \\
\cline { 2 - 5 } & $\mathbf{r}$ & $\mathbf{p}$ & $\mathbf{r}$ & $\mathbf{p}$ \\
\hline Resistência aeróbica & $-0,260$ & 0,929 & $-0,108$ & 0,702 \\
Resistência muscular localizada & 0,364 & 0,201 & 0,040 & 0,889 \\
Flexibilidade & $0,606^{*}$ & 0,022 & $-0,306$ & 0,267 \\
Velocidade & 0,242 & 0,404 & $-0,250$ & 0,370 \\
Potência de membros superiores & 0,322 & 0,262 & 0,343 & 0,211 \\
Potência de membros inferiores & 0,307 & 0,285 & 0,349 & 0,202 \\
Agilidade & 0,221 & 0,448 & $-0,037$ & 0,895 \\
\hline Força dos Processos de Inibição & & \\
\hline Componentes da aptidão física & Homens & & Mulheres \\
\cline { 2 - 5 } & $\mathbf{r}$ & $\mathbf{p}$ & $\mathbf{r}$ & $\mathbf{p}$ \\
\hline Resistência aeróbica & $-0,286$ & 0,322 & 0,112 & 0,692 \\
Resistência muscular localizada & $-0,066$ & 0,824 & $-0,043$ & 0,879 \\
Flexibilidade & 0,213 & 0,465 & $-0,242$ & 0,384 \\
Velocidade & 0,181 & 0,536 & $-0,069$ & 0,333 \\
Potêncilidade & 0,361 & 0,205 & 0,155 & 0,581 \\
\hline Potência de membros superiores & 0,211 & 0,468 & $0,590^{*}$ & 0,021 \\
\hline Po membros inferiores & $-0,830$ & 0,778 & $-0,327$ & 0,234 \\
\hline
\end{tabular}




\section{QUADRO 2}

CORRELAÇÃO ENTRE AS PTSN (MOBILIDADE E EQUILÍBRIO) E ESCORES DOS TESTES FÍSICOS.

\section{Mobilidade}

\begin{tabular}{ccccc}
\hline \multirow{2}{*}{ Componentes da aptidão física } & \multicolumn{2}{c}{ Homens } & \multicolumn{2}{c}{ Mulheres } \\
\cline { 2 - 5 } Resistência aeróbica & $\mathbf{p}$ & $\mathbf{p}$ & 0,098 \\
\hline Flexibilidade & $-0,151$ & 0,605 & $-0,443$ & 0,170 \\
Velocidade & 0,332 & 0,247 & $-0,374$ & 0,574 \\
Resistência muscular localizada & 0,493 & 0,073 & $-0,158$ & 0,120 \\
Potência de membros superiores & 0,412 & 0,143 & 0,419 & 0,269 \\
Aggilidade & 0,102 & 0,727 & 0,305 & 0,255 \\
\hline
\end{tabular}

Equilíbrio

\begin{tabular}{ccccc}
\hline \multirow{2}{*}{ Componentes da aptidão física } & \multicolumn{2}{c}{ Homens } & \multicolumn{2}{c}{ Mulheres } \\
\cline { 2 - 5 } Resistência aeróbica & $\mathbf{r}$ & $\mathbf{p}$ & $\mathbf{r}$ \\
\hline Flexibilidade & 0,323 & 0,260 & $-0,217$ & 0,438 \\
Velocidade & 0,526 & 0,053 & 0,111 & 0,793 \\
Potência de membros superiores & 0,483 & 0,810 & $-0,074$ & 0,978 \\
Potência de membros inferiores & $-0,710$ & 0,056 & 0,008 & 0,517 \\
Agilidade & 0,116 & 0,809 & 0,182 & 0,294 \\
\hline
\end{tabular}




\section{QUADRO 3}

CORRELAÇÃO ENTRE OS TRAÇOS DE PERSONALIDADE (EXTROVERSÃO-INTROVERSÃO E INSTABILIDADE-ESTABILIDADE EMOCIONAL) E OS ESCORES DOS TESTES FÍSICOS.

\section{Extroversão-Introversão}

\begin{tabular}{ccccc}
\hline \multirow{2}{*}{ Componentes da aptidão física } & \multicolumn{2}{c}{ Homens } & \multicolumn{2}{c}{ Mulheres } \\
\cline { 2 - 5 } Resistência aeróbica & $\mathbf{r}$ & $\mathbf{p}$ & $\mathbf{p}$ \\
\hline Fesistência muscular localizada & 0,129 & 0,661 & $-0,202$ & 0,280 \\
Velocidade & 0,259 & 0,371 & 0,299 & 0,937 \\
Potência de membros superiores & 0,232 & 0,424 & $-0,022$ & 0,712 \\
Potência de membros inferiores & $-0,197$ & 0,971 & $-0,104$ & 0,812 \\
Agilidade & 0,045 & 0,499 & 0,067 & 0,194 \\
\hline
\end{tabular}

\section{Estabilidade-Instabilidade Emocional}

\begin{tabular}{ccccc}
\hline Componentes da aptidão física & \multicolumn{2}{c}{ Homens } & \multicolumn{2}{c}{ Mulheres } \\
\cline { 2 - 5 } Resistência aeróbica & $\mathbf{r}$ & $\mathbf{p}$ & $\mathbf{p}$ \\
\hline Fesistência muscular localizada & $-0,054$ & 0,854 & $-0,128$ & 0,981 \\
Velocidade & $-0,027$ & 0,988 & $-0,007$ & 0,689 \\
Potência de membros superiores & 0,102 & 0,926 & $-0,113$ & 0,709 \\
Potência de membros inferiores & 0,155 & 0,598 & 0,105 & 0,416 \\
Aggilidade & 0,190 & 0,727 & $-0,227$ & 0,078 \\
\hline
\end{tabular}




\section{DISCUSSÃO}

Para Anastasi ${ }^{(17)}$ o físico influencia o comportamento em casos de condições patológicas, problemas estruturais e deficiências sensoriais ou motoras e também pela atuação de estereótipos sociais que poderão influenciar as atitudes, as oportunidades que Ihe são oferecidas e mesmo sua autopercepção, tornando o comportamento gradualmente próximo do exigido pelo estereótipo. Também o comportamento influencia no físico. Comportamentos repetitivos diários poderão refletir nas características físicas e doenças psicossomáticas que são associados à tensão emocional e ansiedade.

Analisando os escores do teste de resistência aeróbica a partir da classificação apresentada por Matsudo (11) verifica-se que os sujeitos do gênero masculino possuem uma boa resistência aeróbica, ao passo que, os sujeitos do gênero feminino dentro da mesma classificação apresentam níveis razoáveis de desempenho. Na avaliação da resistência muscular localizada os dados dos sujeitos do gênero masculino são classificados como possuindo um desempenho acima da média, já os resultados dos sujeitos do gênero feminino são classificados como médios para a idade considerando a classificação proposta por Johnson e Nelson (13).

Os resultados do teste de flexibilidade dos sujeitos de ambos os gêneros apontam para um nível fraco de desempenho para a idade segundo a classificação proposta por Heyward apud Pitanga(18). A literatura é restrita quanto a normas para avaliação dos testes de velocidade, no entanto, de acordo com Pereira e Graup (9) os índices do teste de velocidade alcançados pelos sujeitos da amostra são considerados fracos para ambos os gêneros.

Ao comparar-se o valor médio obtido no teste de potência de membros superiores dos sujeitos investigados com a classificação proposta por Johnson e Nelson ${ }^{(13)}$ para estudantes universitários verifica-se que os acadêmicos investigados possuem nível de desempenho intermediário para ambos os gêneros. Na mensuração da potência de membros inferiores também analisando o desempenho dos sujeitos através dos índices propostos por Johnson e Nelson ${ }^{(13)}$ vê-se que os escores representam um nível fraco de desempenho.

Segundo a TABELA proposta por Johnson e Nelson ${ }^{(13)}$, para a classificação dos resultados do teste de agilidade, os resultados obtidos pelos sujeitos do gênero masculino estão entre os percentis 25-50 de desempenho, já os escores obtidos pelos sujeitos do gênero feminino estão entre os percentis 5-25 de desempenho.

Os índices das peculiaridades tipológicas do sistema nervoso identificado nos sujeitos do gênero masculino se encontram a cima dos valores médios propostos por Kalinine e Giacomini (16) para a população em geral. Os valores das peculiaridades tipológicas do sistema nervoso na população masculina em geral são 58,9 para a FPE, 56,8 para FPI, 60 para MOB e 1,04 para a variável EQU. Ainda segundo Kalinine e Giacomini (16), quando o Equilíbrio (E) for de 0,9 até 1,1 o homem possui sistema nervoso equilibrado, caso dos acadêmicos pesquisados neste estudo.

Os resultados obtidos corroboram com os achados de Saldanha Filho ${ }^{(7)}$ que investigou as peculiaridades tipológicas do sistema nervoso em estudantes universitários. Os valores encontrados pelo autor referentes às peculiaridades tipológicas do sistema nervoso (FPE, FPI, MOB e EQU) foram respectivamente: $61,0 \pm 9,8 ; 63,0 \pm 12,2 ; 62,0 \pm 9,6$ e $0,96 \pm 0,2$ para os sujeitos do gênero masculino e $56,5 \pm 11,6 ; 57,0 \pm 12,4 ; 61,0 \pm 10,6$ e $0,98 \pm 0,2$ para os sujeitos do gênero feminino.

Os dados também são convergentes com a investigação em acadêmicos de Educação Física realizada por Paim ${ }^{(19)}$. Os valores encontrados pela autora referente às peculiaridades tipológicas do sistema nervoso (FPE, FPI, MOB e EQU) foram respectivamente: $64,68 \pm 10,40 ; 64,53 \pm 10,80$;

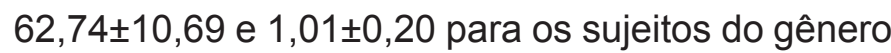


masculino e 58,74 $\pm 11,14 ; 61,11 \pm 9,98 ; 61,61 \pm 10,53$ e $0,97 \pm 0,21$ para os sujeitos do gênero feminino.

$\mathrm{Na}$ TABELA 3 têm-se os resultados da investigação dos traços de personalidade (extroversão-introversão e instabilidade-estabilidade emocional). Os valores dos traços de personalidade são semelhantes ao valor dos universitários investigados por Paim (19) que obteve os seguintes resultados médios: EX-IN 13,51 e I-EE 11,61 em relação aos sujeitos do gênero masculino e EX-IN 12,92 e I-EE 13,30 para os sujeitos do gênero feminino.

Em relação aos traços de personalidade análises com o instrumento Eysenck Personality Inventory (EPI) revelaram que os sujeitos do gênero masculino em termos gerais apresentam tendência à extroversão comparando com as mulheres. São considerados para introversão índices menores e para extroversão índices maiores, tendo como referência média índice equivalente a $12,5^{(8)}$.

Ainda seguindo a classificação do EPI, são considerados para estabilidade emocional índices menores e para instabilidade emocional índices maiores, tendo como referência média 9,0 pontos. Através dessa comparação pôde-se verificar que os índices obtidos no presente estudo encontram-se acima da média considerada por Eysenck, indicando que os acadêmicos do gênero masculino apresentam tendência a Instabilidade emocional e são instáveis em suas emoções, confirmando os dados encontrados por Paim ${ }^{(19)}$.

Pôde-se inferir também que as mulheres apresentam índices de EX-IN com tendência à extroversão. Para I-EE foram encontrados índices maiores que os encontrados para os indivíduos do gênero masculino, indicando traços mais elevados de instabilidade emocional nas mulheres, confirmando os resultados por Paim ${ }^{(19)}$.

No QUADRO 1 foram apresentados os resultados da correlação entre os índices da peculiaridade tipológica do sistema nervoso: Força dos processos de excitação (FPE) e força dos processos de inibição (FPI) e os resultados dos testes físicos. A
FPE, segundo Petrovski ${ }^{20)}$, trata-se do limite da capacidade de trabalho das células nervosas do córtex e do encéfalo, ou seja, a sua capacidade de suportar altos estímulos, sem entrar no estado de inibição. Conforme discute Paim (19) pessoas cujo sistema nervoso tem alto nível da força dos processos de excitação se formam, na maioria dos casos, pessoas corajosas, ativas, extrovertidas e auto-confiantes. Por outro lado, pessoas que têm baixo nível da força dos processos de excitação do sistema nervoso, na maioria dos casos, se tornam introvertidos, melindrosas, pouco ativas e pouco confiantes.

A pessoa com FPI alta, segundo Petrovski (20), caracteriza-se por ter a capacidade de ser discreta em emoções, condutas, ações e reações é um componente necessário na atividade integral e de coordenação do sistema nervoso. Os processos de Inibição juntamente com os processos de excitação asseguram a adaptação do organismo para o ambiente, que está em constante mudança. Quando relacionamos os índices destas variáveis com os testes físicos não foram encontradas correlações estatisticamente significativas para a grande maioria dos testes físicos.

No QUADRO 2 foram mostrados os resultados do teste de correlação entre as peculiaridades mobilidade e equilíbrio com o desempenho nos testes físicos. O nível de mobilidade do sistema nervoso é uma das principais propriedades do sistema nervoso, que consiste na capacidade de reagir rapidamente às mudanças do ambiente ${ }^{(20)}$. O nível de mobilidade dos processos de excitação e inibição que ocorre no sistema nervoso caracteriza a facilidade para passar de uma atividade para a outra com velocidade de adaptação a novas condições.

O equilíbrio dos processos de excitação e inibição que ocorrem no sistema nervoso do homem é uma peculiaridade que se revela pela proporção entre os processos de excitação e dos processos de inibição(20). A noção de equilíbrio dos processos nervosos foi introduzida por Pavlov, e foi considerada, 
Rev. Educ. Fís. 2009 Mar: 144: 3-12. Rio de Janeiro - RJ - Brasil

como uma das independentes peculiaridades do sistema nervoso e, formando junto com as outras peculiaridades do sistema nervoso (força e mobilidade), o tipo de atividade nervosa superior. Segundo Kalinine e Giacomini (16), o valor médio para indivíduos não atletas é 1,04. Segundo estes autores se o equilíbrio for de 0,9 até 1,1 o homem possui sistema equilibrado. Se E > 1,1 o homem possui sistema nervoso desequilibrado no lado da prevalência dos processos de excitação. Através do QUADRO 2 verificou-se que também não houve correlação estatisticamente significativa na maioria dos testes físicos com essas Peculiaridades.

No QUADRO 3 têm-se os resultados da correção dos Traços de Personalidade Extroversão-Introversão (EX-IN) e instabilidade-estabilidade emocional (I-EE). Segundo Eysenck ${ }^{(4)}$, as bases biológicas para a tipologia EX-IN é descrita da seguinte forma: os introvertidos se caracterizam por alta estimulação cortical, o que faz com que estes formam melhores respostas condicionadas. Já com os extrovertidos, o processo é inverso, ou seja, estes condicionam muito pouco, porém, o comportamento socializado, segundo o autor, se deve largamente a respostas condicionadas adquiridas, sob o pretexto da consciência, quando crianças; por conseguinte, os extrovertidos, podem ser encontrados padrões de comportamento não muito aceitos pela sociedade. Através dos resultados apresentados no QUADRO 3 verificou-se que não houve correlação estatisticamente significativa entre os traços de personalidade e o desempenho nos testes físicos.

Aliteratura aponta para diferenças de personalidade na comparação de atletas e não-atletas ${ }^{(21)}$. Além disso, altas pontuações em força de excitação, força de inibição e mobilidade estão relacionadas com elevada auto-estima e baixos índices de vulnerabilidade, desajustamento psicossocial, ansiedade e depressão(22) o que poderia contribuir para maior inserção em atividades esportivas proporcionando maior desenvolvimento das habilidades motoras. No entanto, a ausência de correlação entre as variáveis físicas e psicológicas corroboram com estudo similar realizado em atletas ${ }^{(5)}$ e mostram um comportamento complexo entre essas variáveis em cada indivíduo. Esses resultados ganham importância considerando que acadêmicos de educação física são avaliados durante o curso de formação, considerando, além de suas capacidades cognitivas, o seu desempenho físico em atividades atléticas.

\section{CONCLUSÃO}

A amostra mostrou-se homogênea considerando os indicadores físicos e psicológicos avaliados. Os resultados dos testes físicos demonstraram um nível regular de aptidão com índices mais elevados nos testes que se referem à resistência muscular localizada e resistência cardiorrespiratória para os sujeitos do gênero masculino. A análise psicológica mostrou índices de peculiaridades tipológicas do sistema nervoso mais elevados que a população em geral e os acadêmicos apresentaram uma tendência a extroversão e a instabilidade emocional, principalmente nos sujeitos do gênero feminino.

Não foram encontradas correlações entre os índices das peculiaridades tipológicas do sistema nervoso e dos traços de personalidade com o desempenho físico nos sujeitos investigados. Sugere-se desta forma, que as escolas formadoras destes profissionais atentem para as características tanto físicas como psicológica dos acadêmicos de Educação Física criando métodos de ensino e avaliação condizentes com as características de seus alunos não submetendo-os à práticas corporais além de suas possibilidades individuais.

\section{REFERÊNCIAS BIBLIOGRÁFICAS}

1. Ito PCP, Guzzo RSL. Temperament: characteristics and genetic determination. Psicol Reflex Crit 2002;15(2):425-36.

2. Allport GW. Personalidade padrões e desenvolvimento. São Paulo: Editora da Universidade de São Paulo, 1966. 
12 Rev. Educ. Fís. 2009 Mar: 144: 3-12. Rio de Janeiro - RJ - Brasil

3. Pavlov, IP. Pavlov: psicologia. São Paulo: Ática, 1979.

4. Eysenck HJ. Manual for the Eysenck Personality Inventori. San Diego: Educational and Industrial Testing Service, 1968.

5. Pereira EF, Villis JMC, Paim MCC. Avaliação física e psicológica em atletas de orientação. Revista de Educação Física 2006;134:10-21.

6. Pereira EF, Teixeira CS. Proposta de valores normativos para avaliação da aptidão física em militares da Aeronáutica. Rev Bras Educ Fís Esp 2006;20(4):249-56.

7. Saldanha Filho MF. Investigação da relação entre índices de liderança e peculiaridades tipológicas do sistema nervoso dos estudantes do curso de educação física da universidade Federal de Santa Maria [dissertação mestrado]. Centro de Educação Física e Desportos da Universidade Federal de Santa Maria; 2000.

8. Paim MCC, Kalinine I. Força dos processos de excitação do sistema nervoso e traço de personalidade dos acadêmicos de educação física da Universidade Federal de Santa Maria (UFSM). Rev Educ Fís UEM 2002;13(1):63-69.

9. Pereira EF, Graup S. Aptidão física relacionada à saúde e ao desempenho atlético de calouros de Educação Física. Lecturas Educación Física y Deportes 2007;11. Disponível em URL: http://www.efdeportes.com/efd104/aptidao-fisica. htm (2008 Jan 15)

10. American Alliance for Health, Physical Education, Recreation and Dance. Health related physical fitness test manual. Reston, VA, 1980.

11. Matsudo VKR. Testes em Ciências do Esporte. 7th ed. Londrina: MIDIOGRAF, 2005.

12. Alliance for Health, Physical Education, Recreation and Dance. Youth Fitness Test Manual. Reston, VA, 1976.

13. Johnson BL, Nelson JK. Pratical measurements for evaluation in physical education. 4th ed. Edina: Burgerss Publishing, 1986.

14. Strelau J. O papel do temperamento no desenvolvimento psíquico. Moscou: Progress, 1982.

15. Kalinine I, Giacomini LC, Augusti A. Investigação das peculiaridades tipológicas do sistema nervoso dos atletas jovens de handebol (masculino) vencedores do IV Jogos da Juventude do Brasil. Rev Bras Cienc Esp 2000;21(2/3):24-8.
16. Kalinine I, Giacomini LC. Pesquisa da tipologia dos atletas de alto rendimento no Brasil. Revista Kinesis 1998;20:69-76.

17. Anastasi A. Psicologia Diferencial. São Paulo: Editora Pedagógica e Universitária da Universidade de São Paulo, 1967.

18. Pitanga FJG. Testes, Medidas e Avaliação em Educação Física e Esportes. 2nd ed. Salvador: Edição do Autor, 2001.

19. Paim MCC. Relação entre as peculiaridades tipológicas do sistema nervoso e traços de personalidade [dissertação mestrado]. Santa Maria: Centro de Educação Física e Desportos da Universidade Federal de Santa Maria; 2002.

20. Petrovski AV. Dicionário psicológico breve. Moscou: Polotisdat, 1985.

21. Bara Filho MG, Ribeiro LCS, Garcia FG. Comparação de características da personalidade entre atletas brasileiros de alto rendimento e indivíduos não-atletas. Rev Bras Med Esporte 2005;11(2):115-20.

22. Ito PCP, Gobitta M, Guzzo RSL. Temperamento, neuroticismo e auto-estima: estudo preliminar. Estud Psicol (Campinas) 2007;24(2):143-53.

\section{Endereço:}

Rua: Otacílio Chaves, 253.

Nossa Senhora do Perpétuo Socorro.

CEP 97045-360 - Santa Maria - RS

e-mail: ericofelden@yahoo.com.br 